\title{
Performance of UV pasteurization with quartz glass sleeve on physicochemical properties and microbial activity of pineapple juice
}

\begin{abstract}
Quartz glass has characteristics of high purity, high thermal resistance, high resistivity and high dielectric strength. Currently, majority of research in this area is discussing about the UV reactor equipped by a quartz glass sleeve. The aim of this research is to investigate the effect of quartz glass sleeve on the physicochemical properties and microbial activity of pineapple fruit juice during storage. UV-C radiation is one of the nonthermal technologies that have been introduced in the food industry. UV-C has the advantage of no known toxic or significant nontoxic by-products formed during treatment. The UV reactor that is enclosed by quartz glass can maintain the quality of pineapple juice in terms of color ( $\mathrm{L}^{*}$ value, chroma and hue angle), total soluble solids, $\mathrm{pH}$ and ascorbic acid content. All of the physicochemical properties of pineapple juice underwent significant changes with storage time. Moreover, the UV reactor that is enclosed by quartz glass is able to extend the shelf life of juice for up to 5 weeks and achieve a $5 \log$ reduction of Salmonella typhimurium in the juice after treatment.
\end{abstract}

Keyword: Quartz glass sleeve; Pineapple juice; UV pasteurization 